\title{
NOTE ON SPIN RELAXATION OF THE ISING CHAIN
}

\author{
B. U. FeLderhoF \\ Institute for Theoretical Physics, Rijksuniversiteit, Utrecht \\ (Received February 27, 1971)
}

This note corrects an inaccuracy in our previous paper: 3. U. Felderhof, Reports Math. Phys. 1 (1970), 215.

We would like to correct an inaccuracy in our article. In equation (6.4) the definition of the operators should be modified as follows

$$
\begin{aligned}
& \zeta_{q}^{\dagger}(\beta)=\rho_{ \pm}^{-\frac{1}{2}} \xi_{q}^{\dagger} \rho_{ \pm}^{\frac{1}{2}}, \\
& \zeta_{q}(\beta)=\rho_{ \pm}^{-\frac{1}{2}} \xi_{q} \rho_{ \pm}^{\frac{1}{2}},
\end{aligned}
$$

where the upper sign + should be employed for the $q$-values (4.13a) and the lower sign for the $q$-values (4.13b). The operators $\rho_{ \pm}$are defined by

$$
\rho_{ \pm}=\exp \left[-\beta \mathscr{H}_{N}^{ \pm}\right] / Z_{N}^{ \pm}(\beta),
$$

where the commuting operators $\mathscr{H}_{N}^{+}$and $\mathscr{H}_{N}^{-}$are given in (6.5) and $Z_{N}^{ \pm}(\beta)$ are the corresponding partition functions. With the definition (N.1) the expressions (6.16) remain valid but lead to the conclusion that the state $|0\rangle$ is not the vacuum state for $\xi_{q}$-particles with $q$-values (4.13b). Rather one has

$$
\begin{array}{rll}
\check{\zeta}_{q}|0\rangle=0 & \text { for } & q= \pm(2 m-1) \pi / N, \\
\dot{\zeta}_{q}\left|0^{-}\right\rangle=0 & \text { for } & q= \pm 2 m \pi / N,
\end{array}
$$

where

$$
\left|0^{-}\right\rangle=C|0\rangle
$$

with

$$
\begin{aligned}
C & =\rho_{-}^{\frac{1}{2}} \rho_{+}^{-\frac{1}{2}}=A_{N}\left[\cosh \beta J+\left(c_{N}^{\dagger}-c_{N}\right)\left(c_{1}^{\dagger}+c_{1}\right) \sinh \beta J\right], \\
A_{N} & =\left[Z_{N}^{+}(\beta) / Z_{N}^{-}(\beta)\right]^{\frac{1}{2}}=\left(1+a^{N}\right)^{\frac{1}{2}}\left(1-a^{N}\right)^{-\frac{1}{2}}, \\
a & =\tanh \beta J .
\end{aligned}
$$


Using (4.5) one has

$$
\left|0^{-}\right\rangle=A_{N}\left(\cosh \beta J-\sigma_{N}^{z} \sigma_{1}^{z} \sinh \beta J\right)|0\rangle
$$

and one easily shows

$$
\left\langle 0^{-} \mid 0^{-}\right\rangle=1, \quad\left\langle 0 \mid 0^{-}\right\rangle=A_{N}\left(1+a^{N}\right)^{-1}(\cosh \beta J)^{-1} .
$$

As a consequence of (N.2b) the excited states $\left|q_{k}\right\rangle$, in case $q_{k} \equiv\left(q_{1}, q_{2}, \ldots, q_{k}\right)$ is a set of $q$-values (4.13b), must be defined with respect to the vacuum state $\left|0^{-}\right\rangle$rather than $|0\rangle$. Correspondingly, in this case in (8.5) and (8.9) the state $|0\rangle$ should be replaced by $\left|0^{-}\right\rangle$. For $k$ odd, $\left|\boldsymbol{q}_{k}\right\rangle$ is an eigenstate of $W(\beta)$. In contradiction to the statement following (4.25) we now have

$$
\begin{aligned}
& W^{+}(\beta)|0\rangle=0, \\
& W^{-}(\beta)\left|0^{-}\right\rangle=0 .
\end{aligned}
$$

With the above interpretation of the states $\left|\boldsymbol{q}_{k}\right\rangle$ the remaining formalism needs no alteration.

\section{Acknowledgment}

I am grateful to Mr. H. J. Hilhorst for pointing out this error. 